\title{
SOME IMPLICATIONS OF WORK HARDENING AND IDEAL PLASTICITY*
}

\author{
BY \\ D. C. DRUCKER \\ Brown University
}

1. Summary. The purpose of this note is to point out the severe restriction imposed on possible stress-strain relations by a mathematical formulation of the concepts of work hardening and ideal plasticity. Using this condition, an algebraic derivation and extension is given of Prager's extension ${ }^{1}$ of the Mises plastic potential function. A brief discussion is also given of the meaning of stability of plastic deformation as contrasted with stability of non-conservative systems in general.

2. Introduction. A mathematical theory of plasticity must of necessity be based upon simplifying assumptions. For example, a good first approximation may be obtained by ignoring time effects in most structural metals at room temperature under isothermal conditions. A stress-strain diagram, therefore, represents a succession of states of static equilibrium. Furthermore, it is an experimental fact that metals generally work harden; then each equilibrium state is said to be stable. The phenomenon of the upper yield point, exhibited by a few metals, notably mild steel, is an important exception. Another exception is the region beyond the ultimate strength on a nominal tensile stress-strain plot when necking occurs, Fig. 1. However, this real instability is in terms of force; the actual stress continues to increase until failure occurs.

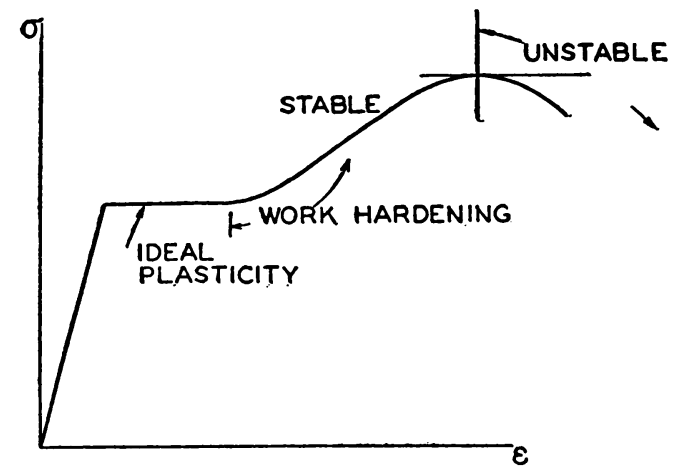

Fig. 1. Plastic deformation.

3. Work hardening. The meaning of work hardening in simple tension is just that stress is a monotonically increasing function of strain, Fig. 1. The plastic deformation is then said to be stable. For more general states of stress and paths of loading no such simple picture can be drawn. The concept of work hardening, or stability in a restricted sense, can be expressed in terms of the work done by an external agency which slowly applies an additional set of stresses and then slowly removes them. The original con-

*Received Feb. 21, 1949. The conclusions presented here were obtained in the course of research conducted under Contract N70nr-358 sponsored jointly by the Office of Naval Research and the Bureau of Ships.

${ }^{1} \mathrm{~W}$. Prager, Recent developments in the mathematical theory of plasticity, presented to the 7th Internat. Congress for Appl. Mech., London, 1948, J. Appl. Phys. 20, 235-241 (1949). 
figuration, or state of strain, may or may not be restored. This external agency is to be understood as entirely separate and distinct from the agency which causes the existing state of stress and which has produced the existing state of strain.

Work hardening implies that for all such added sets of stresses the material will remain in equilibrium and further that

a) positive work is done by the external agency during the application of the set of stresses, and

b) the net work performed by it over the cycle of application and removal is zero or positive.

It should be emphasized that the work referred to is not the total work done by all of the forces acting, it is only the work done by the added set on the displacements which result. Rephrased, work hardening means that useful net energy over and above the elastic energy can not be extracted from the material and the system of forces acting upon it.

Consider a unit volume of material in which there is a homogeneous state of stress $\sigma_{i j}$ and strain $\epsilon_{i j}$. Suppose an external agency to apply small surface tractions which alter the stress at each point by $d \sigma_{i j}$, and produce small strain increments $d \epsilon_{i j}$. Next suppose the external agency to remove these added tractions, thus releasing the elastic strain increments $d \epsilon_{i j}^{e}$. It then follows from condition (a) that (repeated subscripts denote summation) $d \sigma_{i j} d \epsilon_{i j}$ is positive and from (b) that $d \sigma_{i j}\left(d \epsilon_{i j}-d \epsilon_{i j}^{e}\right)$ is either positive or zero. Since the plastic strain increments $d \epsilon_{i j}^{p}=d \epsilon_{i j}-d \epsilon_{i j}^{e}$,

$$
\begin{aligned}
& d \sigma_{i j}\left(d \epsilon_{i j}^{o}+d \epsilon_{i j}^{p}\right)>0, \\
& d \sigma_{i j} d \epsilon_{i j}^{p} \geq 0 .
\end{aligned}
$$

An equivalent manner ${ }^{2}$ of specifying work hardening which leads to (1) and (2) is that as any set of strain increments is increased in ratio from any initial state of stress and strain, more and more total and also plastic work per unit volume is required per given change in any one of the components of strain.

4. Stress-strain relations (work hardening). In this section, two basic assumptions will be made for a work hardening material.

1. A loading function exists. At each stage of a plastic deformation a function $f\left(\sigma_{i j}\right)$ exists such that further plastic deformation takes place only for $f\left(\sigma_{i j}\right)>k$. Both $f$ and $k$ may depend upon the state of plastic strain and the plastic strain history.

2. The relation between the infinitesimals of stress and strain is linear. The sum of the increments in plastic deformation, obtained separately from each of two sets of infinitesimal stress increments $d \sigma_{k l}^{\prime}$ and $d \sigma_{k l}^{\prime \prime}$, neither of which constitutes unloading, is the same as the plastic deformation resulting from

$$
d \sigma_{k l}=d \sigma_{k l}^{\prime}+d \sigma_{k l}^{\prime \prime}, \quad\left(\partial f / \partial \sigma_{k l}\right) d \sigma_{k l}^{\prime} \geq 0, \quad\left(\partial f / \partial \sigma_{k l}\right) d \sigma_{k l}^{\prime \prime} \geq 0
$$

Following the geometric proof given by Prager, ${ }^{1}$ it is necessary to show that any $d \sigma_{k l}$ representing loading, $\left(\partial f / \partial \sigma_{k l}\right) d \sigma_{k l}>0$, may be decomposed into $d \sigma_{k l}^{\prime}$ and $d \sigma_{k l}^{\prime \prime}$,

${ }^{2} \mathrm{D}$. C. Drucker, The significance of the criterion for additional plastic deformation of metals, presented at the 1948 Annual Meeting of the Society of Rheology, J. Colloid Sci. 4, 299-311 (1949). 
where the arbitrary choice is made that $d \sigma_{k l}^{\prime}$ produces no plastic deformation, and $d \sigma_{k l}^{\prime \prime}$ is proportional to the gradient of $f$ :

$$
\begin{aligned}
& \frac{\partial f}{\partial \sigma_{k l}} d \sigma_{k l}^{\prime}=0 ; \\
& \frac{\partial f}{\partial \sigma_{k l}} d \sigma_{k l}^{\prime \prime}=\frac{\partial f}{\partial \sigma_{k l}} d \sigma_{k l} ; \quad d \sigma_{k l}^{\prime \prime}=r \frac{\partial f}{\partial \sigma_{k l}}, \quad r>0 .
\end{aligned}
$$

This can always be done as $\left(\partial f / \partial \sigma_{k l}\right) d \sigma_{k l}^{\prime \prime}=r\left(\partial f / \partial \sigma_{k l}\right)\left(\partial f / \partial \sigma_{k l}\right)>0$ and can be made equal to $\left(\partial f / \partial \sigma_{k l}\right) d \sigma_{k l}$ by a proper choice of $r$,

$$
r=\frac{\left(\partial f / \partial \sigma_{k l}\right) d \sigma_{k l}}{\left(\partial f / \partial \sigma_{m n}\right)\left(\partial f / \partial \sigma_{m n}\right)} .
$$

The linearity assumption states further that if all $d \sigma_{k l}$ are increased in ratio, all $d \epsilon_{i j}^{p}$ are increased in the same proportion, so that

$$
d \epsilon_{i j}^{p} \sim r
$$

or

$$
d \epsilon_{i j}^{p}=g_{i j} \frac{\partial f}{\partial \sigma_{k l}} d \sigma_{k l},
$$

where the $g_{i j}$ may depend upon stress, strain, and their history but do not involve $d \sigma_{k l}$.

Using the work hardening condition, we can write Eq. (2) as

$$
0 \leq d \sigma_{i j} d \epsilon_{i j}^{p}=d \sigma_{i j}^{\prime} d \epsilon_{i j}^{p}+d \sigma_{i j}^{\prime \prime} d \epsilon_{i j}^{p},
$$

for any $d \sigma_{i j}^{\prime}$ such that $\left(\partial f / \partial \sigma_{i j}\right) d \sigma_{i j}^{\prime}=0$, and for $d \sigma_{i j}^{\prime \prime}$ producing $d \epsilon_{i j}^{p}$. Since the relationship $d \sigma_{i j}=C d \sigma_{i j}^{\prime}+d \sigma_{i j}^{\prime \prime}$ also constitutes loading for all values of $C$, whether positive or negative, and produces the same $d \epsilon_{i j}^{p}$, the work hardening condition becomes

$$
C d \sigma_{i j}^{\prime} d \epsilon_{i j}^{p}+d \sigma_{i j}^{\prime \prime} d \epsilon_{i j}^{p} \geq 0 .
$$

The term $C d \sigma_{i j}^{\prime} d \epsilon_{i j}^{p}$ is the work done on the plastic strain increments by a set of stress increments which produce no plastic strain. Its magnitude must be zero, for if it were not, a $C$ could be chosen of proper sign and magnitude to violate (6).

Therefore,

$$
d \sigma_{i j}^{\prime} d \epsilon_{i j}^{p}=d \sigma_{i j}^{\prime} g_{i j} \frac{\partial f}{\partial \sigma_{k l}} d \sigma_{k l}=0
$$

or

$$
d \sigma_{i j}^{\prime} g_{i j}=0,
$$

where

$$
\frac{\partial f}{\partial \sigma_{i j}} d \sigma_{i i}^{\prime}=0 .
$$

Conditions (7) must be satisfied for all $d \sigma_{i j}^{\prime}$ satisfying (3) so that

$$
g_{i i}=G \frac{\partial f}{\partial \sigma_{i j}},
$$


where $G$ is a scalar which may depend upon stress, strain, and history. The only permissible stress-strain relation consistent with the two basic assumptions made at the beginning of this section is, therefore,

$$
d \epsilon_{i j}^{p}=G \frac{\partial f}{\partial \sigma_{i i}} \frac{\partial f}{\partial \sigma_{k l}} d \sigma_{k l} .
$$

It is of interest to note that the work hardening condition (a) automatically insures that unique increments of stress in the interior of a body result from given increments in surface traction ${ }^{1}$, or, more generally, that the history of the state of stress at each interior point is uniquely determined by the history of the surface loading.

5. Ideal plasticity. Ideal plasticity in simple tension is indicated by a horizontal line on the stress-strain diagram, Fig. 1. The stress remains constant as the strain increases. For more general paths of loading, ideal plasticity means that the work done by an external agency which slowly applies and then removes a set of stresses is zero over an equilibrium cycle. Equation (2), for a work hardening material, is replaced by

$$
d \sigma_{i j} d \epsilon_{i j}^{p}=0
$$

for an ideally plastic one. The equality in Eq. (2) applies only when $d \epsilon_{i j}^{p}=0$ so that the difference is by no means trivial.

6. Stress-strain relations (ideal plasticity). The further assumption of ideal plasticity is that $f\left(\sigma_{i i}\right)$, a function of stress alone, exists such that plastic deformation takes place without limit when $f\left(\sigma_{i i}\right)=k$ and that the material is elastic for $f\left(\sigma_{i j}\right)<k$. During flow, therefore,

$$
\frac{\partial f}{\partial \sigma_{i j}} d \sigma_{i j}=0 \text {. }
$$

As all $d \sigma_{i i}$ satisfying Eq. (11) must also satisfy Eq. (10),

$$
d \epsilon_{i i}^{p}=\lambda \frac{\partial f}{\partial \sigma_{i j}},
$$

where $\lambda$ is a scalar. The form of $f$ may be as anisotropic as desired and of any degree in the components of stress.

7. Stability of non-conservative systems. When a metal work hardens, the plastic

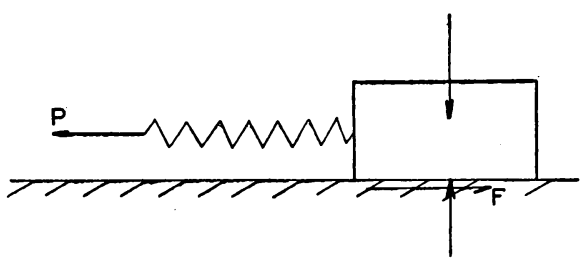

FIG. 2. A simplified model of plastic flow. The coefficient of friction is variable for a work hardening material. Here $d \sigma_{i j} d \epsilon^{p}{ }_{i j} \geq 0$ is required.

deformation is termed stable. The question arises as to whether this is stability in the conventional sense. Figures 2 and 3 show that work hardening conditions (a) and (b) do imply stability, while Figs. 4 and 5 show that the converse is not true. 
Suppose, in Fig. 2, that $P$ is considerably less than the maximum permissible frictional force $F$ (conservative system case). Application of a small positive or negative increment of force $\Delta P$ will produce a displacement of the end of the spring in the direction of $\Delta P$. The external agency adding $\Delta P$ will do positive work in each case and will

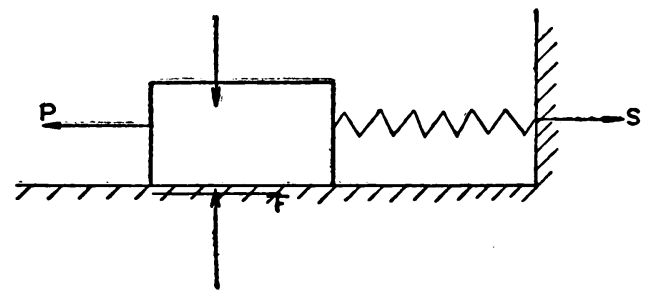

FIG. 3. A model in which the elastic strain is not recoverable directly. Here $d \sigma_{i j}\left(d \epsilon^{e}{ }_{i j}+d \epsilon^{p}{ }_{i j}\right) \geq$ 0 is required.

do zero net work over a cycle of application and removal of $\Delta P$. For a conservative system, work hardening condition (a) is equivalent to a minimum energy principle. Although not useful for such a system, condition (b) is always satisfied, as the net work is zero.

Now let $P$ equal the maximum permissible value of $F$ for the position of the block, and further suppose that the coefficient of friction will increase as the block moves to the left. Under a decrement of force, the system acts in a conservative manner. However, an increment $\Delta P$ will stretch the spring and also move the block; for both reasons, $\Delta P$ will do positive work. Removal of $\Delta P$ releases the elastic energy increment, but the block remains in place. Consequently, the net work done by the external agency over the cycle is positive. The system is obviously stable under disturbances $\Delta P$; an input of energy is required to displace the block. If the coefficient of friction decreases as the block moves to the left, negative $\Delta P$ is required to maintain equilibrium when the motion occurs. The work done by $\Delta P$ is negative, and the system is clearly unstable. In this case, an equilibrium cycle is not possible unless the friction coefficient should increase again, as the motion proceeds.

The system of Fig. 3 gives comparable but somewhat different results. Suppose $P$ to be equal to the sum of the maximum frictional force $F$ for the position of the block and the spring force $S$. If $F$ remains fixed or increases with displacement to the left, an increment $\Delta P$ in $P$ does positive work. On removal of $\Delta P$, the elastic energy increment remains in the spring. Work hardening conditions (a) and (b) are satisfied, and the system is clearly stable under possible disturbances $\Delta P$; an input of energy is required for motion. On the other hand, if $F$ should decrease more than $S$ increases, the system is unstable. Work done by the external agency during the application of the additional force required for equilibrium and over the complete cycle is negative.

Figures 2 and 3 illustrate the sufficiency of conditions (a) and (b) for stability of the usual type; no displacement from the equilibrium position without an input of work. They are one-dimensional examples, however, and the analogy between $P$ and stress and between displacement and strain is really not so close.

Figure 4 brings out the dissimilarity and also the more general problem of stability. If $V$ remains constant, Fig. 4 reduces to Fig. 2. However, if $P=\mu N$ and $V$ is increased 
by $\Delta V, P$ must be decreased by $\mu \Delta V$ to maintain equilibrium. Positive work is done in the application of $\Delta V$ and $-\mu \Delta V$. If these added forces are removed in ratio, the total work over the cycle is zero. If $\Delta V$ is removed first, the result is the same; but if $-\mu \Delta V$, the decrement in $P$, is taken away first, the block will displace to the left and

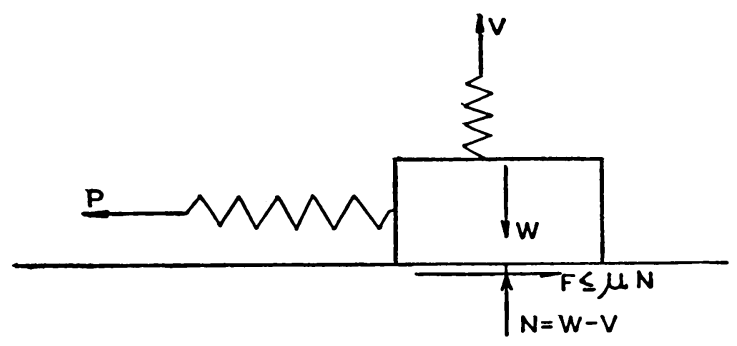

FIG. 4. Work can be extracted.

negative work will be done by the external agency over the complete cycle of application and removal of the additional forces. Repeated cycles will cause continued displacement of the block if the friction coefficient remains constant, but will produce no additional motion, after the first cycle, if $\mu$ increases. From one point of view the system is un-

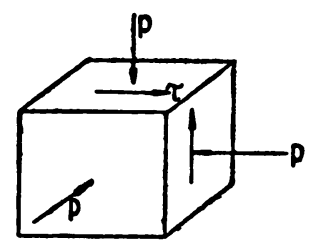

(a)

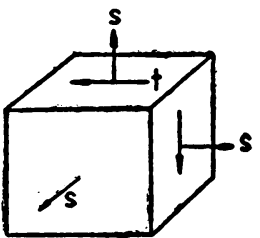

(b)

Fig. 5. Hydrostatic pressure and shear.

stable under the disturbances because the block will move without any external agency doing work on it. More realistically and usefully, if the block moves only infinitesimally for repeated infinitesimal disturbances ( $\mu$ increasing), the system would be considered stable. The case of constant $\mu$ would be termed neutral, and only if finite work could be

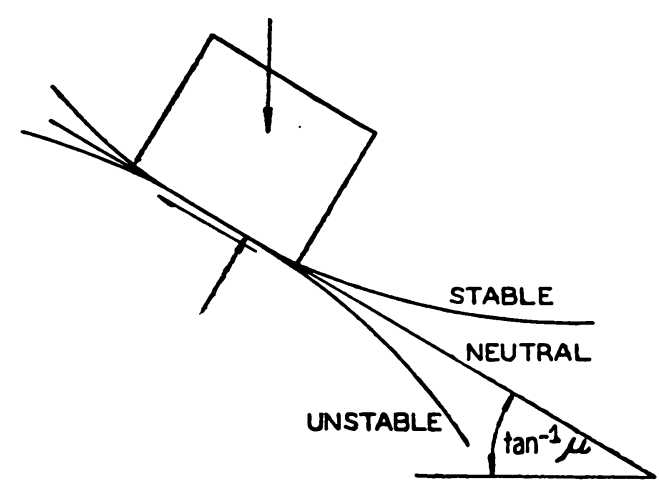

Fig. 6. Stability of non-conservative systems.

extracted for infinitesimal disturbance ( $\mu$ decreasing) would the term unstable be justified in general for a non-conservative system. 
Another simple example of these distinctions is furnished by a block on a surface inclined at the friction angle, Fig. 6. Assuming a constant coefficient of friction, if the surface is a plane, infinitesimal disturbing forces applied to the block normal to the plane will cause the block to move and continue to move down the plane for any finite distance. During this downward motion the block cannot do a finite amount of work on an external system, as the work done by the gravitational force is dissipated by the frictional work. The term neutral seems appropriate. If the surface is curved so that the slope becomes steeper as the block moves, the block will accelerate. Work can, therefore, be done on an external system; for example, a spring may be compressed. The system is unstable. If, on the other hand, the curvature of the surface diminishes the slope, then an infinitesimal disturbance will produce an infinitesimal motion. Repetition of the disturbance will cause no additional motion; the system is clearly stable.

The stability of plastic deformation implied by work hardening is stability in the strictest sense. Even the infinitesimal motion for Fig. 4 with $\mu$ increasing is not permissible. Figure 5 emphasizes this feature for plastic deformation and is a truly critical illustration of the concepts advanced. Suppose Fig. 5a to represent a portion of a work hardening body subjected to a homogeneous state of stress composed of a hydrostatic pressure $p$ and a shearing stress $\tau$. Consider the external agency to apply and then remove a hydrostatic tension $s$ and shearing decrement $t$. As Bridgman has shown, ${ }^{3}$ a high hydrostatic pressure increases the flow stress of metals. For simplicity, assume the yield stress in simple shear at some stage of plastic deformation to be given by

$$
\tau_{y}=\tau_{0}+k p,
$$

where $\tau_{0}$ is some initial value and $k$ is a positive constant. At $p-s$ the yield value will be $\tau_{0}+k p-k s$. Now let $\tau=\tau_{\nu}$ and $t=k s$. The states of stress given by Figs. 5a and also $5 \mathrm{a}$ plus $5 \mathrm{~b}$ are then exactly on the yield or loading surface. If $t$ is removed while $s$ is maintained, the body will deform plastically. Let the added hydrostatic tension be removed next. If the assumption is made that the flow pattern is unaffected by the hydrostatic pressure and is, therefore, simple shear strain, work will be extracted from the system in the cycle. The same result can be obtained directly by taking $s_{1}=s$ but $t_{1}<k s$. When $s_{1}$ and $t_{1}$ are applied simultaneously instead of $s$ and $t$, negative plastic work is done by $t_{1}$, zero plastic work by $s_{1} ; d \sigma_{i j} d \epsilon_{i i}^{p}<0$.

This contradiction to Eq. (2) shows that such a material is analogous to the friction example, Fig. 4, and is not truly work hardening despite the fact that tensile or shear stress-strain curves would look entirely proper. However, the conclusion is not that work hardening metals do not exist but is instead that real metals which do exhibit dependence on the mean normal stress also increase in volume as the plastic deformation proceeds ${ }^{4}$ and that the plastic work done by $s$ or $s_{1}$ is more than that done on $t$ or $t_{1}$ in most, and possibly all, cases of engineering interest.

Taking the loading function $f$ in Eq. (9) as

$$
f=+J_{2}^{1 / 2}+\alpha J_{1}
$$

${ }^{3} \mathrm{P}$. W. Bridgman, Effects of high shearing stress combined with high hydrostatic pressure, Phys. Rev. 48, 825-847 (1935).

${ }^{4}$ A. H. Stang, M. Greenspan, and S. B. Newman, Poisson's ratio of some structural alloys for large strains, J. Research Nat. Bur. Standards 37, 211-221 (1946). 
where $\alpha$ is a constant, $J_{2}=s_{i j} s_{i j} / 2, J_{1}=\sigma_{k k}, s_{i j}=\sigma_{i j}-J_{1} \delta_{i j} / 3$, illustrates but does not, of course, prove this point. Loading, or further plastic deformation, results if

$$
\frac{\partial f}{\partial \sigma_{i j}} d \sigma_{i j}=d\left(J_{2}^{1 / 2}\right)+\alpha d J_{1}>0,
$$

which always insures positive

$$
d \sigma_{i j} d \epsilon_{i j}^{p}=G d \sigma_{i j} \frac{\partial f}{\partial \sigma_{i j}}\left(\frac{\partial f}{\partial \sigma_{k l}} d \sigma_{k l}\right) .
$$

The plastic stress-strain relation is

$$
d \epsilon_{i i}^{p}=G\left(\frac{s_{i i}}{2 J_{2}^{1 / 2}}+\alpha \delta_{i i}\right) d f,
$$

and the plastic dilation is, therefore,

$$
d \epsilon_{k k}^{p}=3 \alpha G d f .
$$

For the loading shown in Fig. 5, the plastic increment in the engineering shearing strain is

$$
d \gamma^{p}=2 d \epsilon_{\tau}^{p}=G d f
$$

so that

$$
d \epsilon_{k k}^{p} / d \gamma^{p}=3 \alpha,
$$

where $3 \alpha$ is the small slope of the straight line relating the yield stress in shear to the mean normal pressure, $k$ in Eq. (13).

The preceding example shows that consistency is obtained and uniqueness of solution assured if the small plastic volume increase which does occur is taken into account. It does not, however, prove that some material system which can do work on an external agent in a closed force cycle, does not, or can not, exist. The argument is only that such a material is not work hardening. Moreover, it is found experimentally in the plastic deformation of most metals in the range of engineering interest that the only energy available is in the form of elastic energy, and that no additional amount of work can be obtained from the system of forces acting on a plastically deformed metal which is in a state of stable equilibrium. No work can be extracted in a force cycle, since the elastic energy is the same at the beginning and the end of the cycle.

8. Conclusion. The usual basic assumptions of the theory of plasticity when coupled with a mathematical formulation of the concepts of work hardening and ideal plasticity require stress-strain relations to be of the Mises-Prager form, $d \epsilon_{i j}^{p}=\lambda\left(\partial f / \partial \sigma_{i j}\right)$. For a work hardening material, $\lambda=G\left(\partial f / \partial \sigma_{k l}\right) d \sigma_{k l}$. $G$ and $f$ may depend upon stress, plastic strain, and the history of plastic strain. 